S. D'Arco, J. A. Suul and J. Beerten, "Analysis of accuracy versus model order for frequencydependent Pi-model of HVDC cables," in Proc. IEEE 17th Workshop on Control and Modeling for Power Electronics (COMPEL) 2016, Trondheim, Norway, 27-30 Jun. 2016, 8 pages.

Digital Object Identifier: $10.1109 /$ COMPEL.2016.7556700

URL:

http://ieeexplore.ieee.org/stamp/stamp.jsp?tp=\&arnumber=7556700\&isnumber $=7556653$

(C) 2016 IEEE. Personal use of this material is permitted. Permission from IEEE must be obtained for all other users, including reprinting/ republishing this material for advertising or promotional purposes, creating new collective works for resale or redistribution to servers or lists, or reuse of any copyrighted components of this work in other works. 


\section{Analysis of Accuracy versus Model Order for Frequency-Dependent Pi-model of HVDC Cables}

\author{
Salvatore D' Arco ${ }^{1}$ \\ ${ }^{1}$ SINTEF Energy Research \\ Trondheim, Norway \\ salvatore.darco@sintef.no
}

\author{
Jon Are Suul ${ }^{1,2}$ \\ 2 Dept. Electric Power Engineering \\ NTNU \\ Trondheim, Norway \\ jon.are.suul@ntnu.no
}

\author{
Jef Beerten ${ }^{3}$ \\ ${ }^{3}$ Dept. Electrical Engineering \\ KU Leuven - EnergyVille \\ Heverlee - Genk, Belgium \\ jef.beerten@esat.kuleuven.be
}

\begin{abstract}
Accurate eigenvalue analysis of High Voltage Direct Current (HVDC) transmission systems depends on state-space models that are accounting for the frequency-dependent characteristics of the dc cables. A classical simplified model with cascaded pi-sections can be enhanced to capture the frequency dependency of cable parameters by adding parallel RL branches for representing the longitudinal impedances. This paper analyzes the performances in terms of accuracy that can be achieved with such a frequency-dependent pi-section (FD- $\pi$ ) representation. The obtained results are used to identify the number of cascaded sections and the number of parallel branches that will ensure a model configuration with the maximum accuracy for a specific model order. Furthermore, the presented analysis is utilized to identify what model order and model configuration are needed for ensuring a specified accuracy over a given frequency range, depending on the cable length. Based on the presented analysis, clear guidelines can be derived for selecting the most suitable model of an HVDC cable with a certain length, depending on the required accuracy and frequency range.
\end{abstract}

Index Terms-Cable modelling, HVDC transmission, vector fitting, small-signal stability, state-space modelling

\section{INTRODUCTION}

Power transmission with HVDC connections based on Voltage Source Converter (VSC) technology is expected to have an increasingly important role in future large-scale power systems [1]. In addition to the initially targeted applications for interconnections in weak grids or between asynchronous systems, the recent advances in efficiency and scalability by the use of Modular Multilevel Converters (MMCs) has made VSC HVDC a viable option for bulk power transfer [2], [3]. Thus, methods for analysis of power system stability should be enhanced to include and correctly account for the dynamics associated with such HVDC systems and their main elements [4], [5]. However, small-signal state-space modelling of VSC HVDC systems, including the emerging MMC topologies and the HVDC cables, is not yet a mature field, and is consequently an active area of present research.

The work of SINTEF Energy Research in this paper was supported by the project "Protection and Fault Handling in Offshore HVDC Grids," (ProOfGrids), financed by the Research Council of Norway's RENERGI program and the industry partners; EDF, National Grid, Siemens, Statkraft, Statnett, Statoil and NVE.

The work of Jef Beerten is funded by a postdoctoral research grant from the Research Foundation Flanders (FWO).

978-1-5090-1815-4/16/\$31.00 (C)2016 IEEE
The correct representation of oscillations and dynamics in a VSC-based HVDC transmission scheme depends on an accurate model of the dc cable or dc line. For time-domain simulations, detailed modelling of travelling wave effects and the frequency-dependent distributed parameter characteristics of transmission lines or cables have been thoroughly addressed in the scientific community. As a result, frequency-dependent wideband models, like the Universal Line Model (ULM) [6], are available in several commercial software packages for time-domain simulations. However, such models are not compatible with the state-space representation needed to assess the small-signal dynamics and stability characteristics by eigenvalue analysis.

For small-signal state-space modelling of VSC HVDC systems, classical cascaded pi-section models of dc cables are commonly applied since they can be easily translated into a state-space model [7], [8], [9], [10], [11]. However, such models fail to capture the frequency-dependent characteristics of the cable parameters, which are essential to account for the damping effects in the system. The limits of the conventional cascaded pi-section representation and the potential impact of ignoring the frequency-dependent characteristics of HVDC cables have recently been highlighted in [12]. Indeed, a higher number of cascaded sections does not necessarily lead to a better accuracy.

An enhanced pi-section model with multiple parallel RL branches in each cascaded section, to account for the frequency-dependency of the cable parameters, was first proposed in [13] and further discussed in [12] and [14]. This frequency-dependent pi-section (FD- $\pi$ ) model can accurately represent the cable characteristics within a limited frequency range, and since its formulation corresponds to a lumped parameter equivalent circuit it can also be directly translated into state-space equations. Thus, the FD- $\pi$ model is inherently suitable for eigenvalue-based stability analysis, although its equivalent circuit can also be used for time-domain simulation when only dynamics in the lower frequency range are of interest.

In general, the accuracy of an FD- $\pi$ model can be enhanced by increasing the number of the cascaded sections and/or the number of parallel branches that together represent two degrees of freedom in the model configuration. 


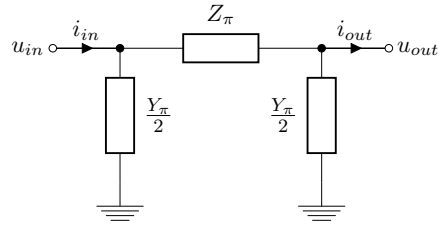

(a)

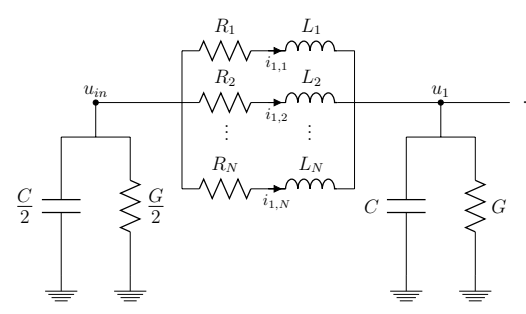

(1)

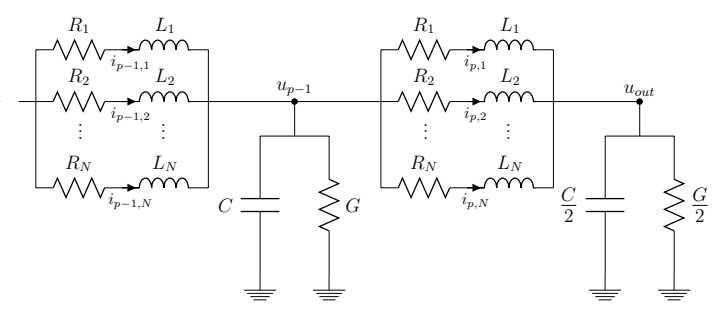

(b)

Fig. 1. a) pi representation of HVDC cable with frequency dependent impedances; b) circuital representation of the FD- $\pi$ model.

However, both a higher number of sections and a higher number of branches will lead to a higher total order for the cable model with obvious consequences in terms of increased complexity and computational requirements. Moreover, for relatively high model orders, the improvements in accuracy when further increasing the number of cascaded sections or parallel branches tend to become almost negligible within the frequency range of interest for small-signal stability analysis of control interactions in HVDC systems. Thus, the selection of the model order should be a balanced trade-off between accuracy and complexity. It should also be noted that multiple configurations (e.g. more cascaded sections but less branches or vice versa) can lead to the same model order, but with different characteristics and hence accuracy.

The impact of selecting different numbers of pi-sections and different numbers of parallel RL-branches in the FD$\pi$ model was illustrated for a specific cable in [12], [13]. However, the studies did not go into full-detail on how the FD$\pi$ model should be configured to ensure a sufficiently accurate cable model while limiting the model order. This paper further analyzes the FD- $\pi$ model from [13] by presenting a systematic analysis of how a change in the number of cascaded sections or the number of parallel branches influences the accuracy of the model. It will also be shown how an optimal configuration ensuring the highest accuracy for a given model order can be determined. Moreover, examples are offered of how the optimal model configuration depends on the cable length and on the required frequency range where the model should ensure a minimum accuracy. The presented analysis can serve as basis for selecting a model configuration suitable for representing a given cable under specific performance requirements.

\section{FREQUENCY-DEPENDENT LUMPED PARAMETER MODEL FOR STATE-SPACE REPRESENTATION}

The behavior of an HVDC cable can be accurately represented in the frequency domain by the circuital equivalent model in Fig. 1a. The series and shunt elements of this model account for the frequency-dependent characteristics of the cable parameters and for the non linear dependence of the cable impedance as a function of the cable length. These circuit elements can be expressed by:

$$
Z_{\pi}(\omega)=z(\omega) \ell \frac{\sinh \gamma(\omega) \ell}{\gamma(\omega) \ell}
$$

$$
\begin{gathered}
Y_{\pi}(\omega)=y(\omega) \ell \frac{\tanh \frac{\gamma(\omega) \ell}{2}}{\frac{\gamma(\omega) \ell}{2}} \\
\gamma(\omega)=\sqrt{z(\omega) y(\omega)}
\end{gathered}
$$

In these equations, $z$ and $y$ are the frequency-dependent cable impedance and admittance per unit length, which also define the propagation constant $\gamma$, and $l$ denotes the cable length.

A circuital representation of the FD- $\pi$ model from [12], [13] is shown in Fig. 1b. For the frequency range of interest in most transmission system analysis, the conductance and capacitance of the cable, determining the shunt admittance $y$, can be assumed constant [15]. By adding several parallel RL branches, this scheme can take into account the frequencydependency for the series impedance $z$ of the cable.

The values for the longitudinal parameters in the model of Fig. 1b should be obtained so that the parallel RL branches can reproduce the frequency characteristic of the series impedance per unit length $z(\omega)$, which is approximated as:

$$
\frac{1}{z(\omega)} \approx \sum_{i=1}^{n} \frac{1}{r_{i}+j \omega l_{i}}
$$

As initially proposed in [13], the longitudinal parameters for the parallel branches can be determined by applying vector fitting [16].

The number of the cascaded sections $n$ and the number of parallel branches together represent two degrees of freedom in the configuration of a FD- $\pi$ model. The total order $N$ for the resulting cable model can be expressed as:

$$
N=n \cdot m+n+1
$$

As an example, the impedance of a $400 \mathrm{~km}$ XLPE cable for VSC HVDC transmission, with data from [17] is shown in Fig. 2. In this figure, the reference model from Fig. 1a is compared to the frequency characteristics of the impedance resulting from the FD- $\pi$ model with a number of cascaded sections $n=10$ and different values of parallel branches $m$. From this figure, it is clearly seen that the traditional pisection model without parallel RL branches results in a poor representation of the internal resonances in the cable, as well as the associated damping. However, already with $m=2$, the FD- $\pi$ model will approximately indicate the 3 to 4 first resonance frequencies of the cable. Increasing the number of 


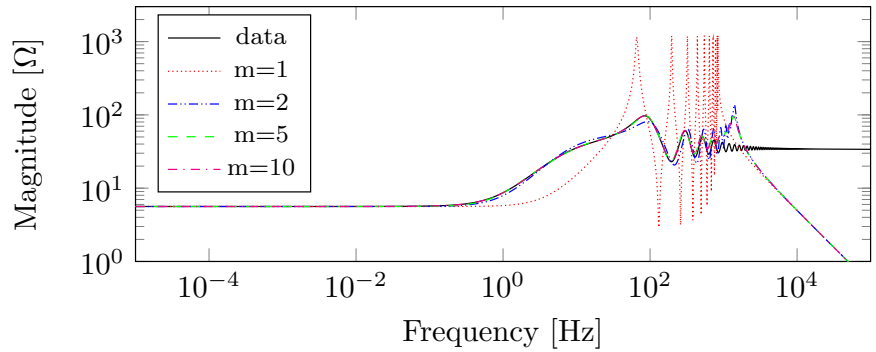

(a)

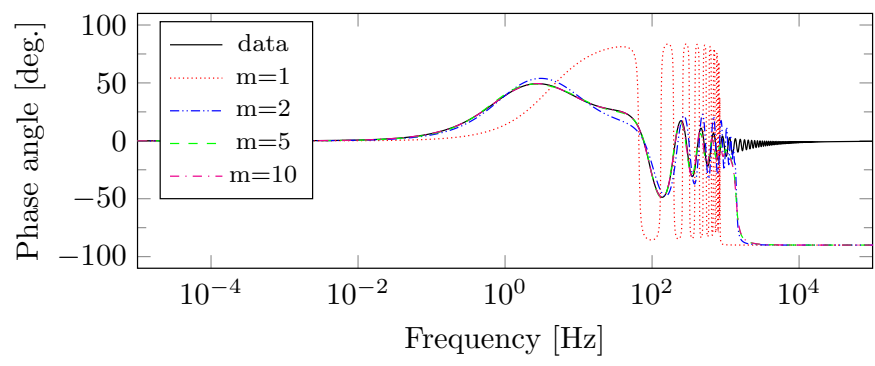

(b)

Fig. 2. Cable impedance comparison of a $400 \mathrm{~km}$ XLPE VSC HVDC cable for different number of parallel branches $m$ (number of pi-sections $n=10$ ).

parallel branches will improve the accuracy, but the figure also indicates that the accuracy of the FD- $\pi$ model of the $400 \mathrm{~km}$ cable will not increase much by adding more than 5 parallel branches.

\section{DEFINITION OF ACCURACY METRICS IN THE FREQUENCY DOMAIN}

In general, the use of multiple parallel branches in the FD$\pi$ model is suitable for representing the frequency-dependent behavior of the cable within the lower frequency range. However, independently from the number of parallel branches or pi sections, the FD- $\pi$ model has a natural limit for representation of the cable at very high frequencies. This can be noticed in Fig. 2, where the amplitude of the FD- $\pi$ model decays linearly in the logaritmic scale at high frequencies while the amplitude of the reference model tends asymptotically to a constant. Thus, the evaluation of the deviations between the reference model and an FD- $\pi$ model should be referred to the range below an upper frequency limit where the accuracy of the model is of importance. This is not a significant limitation of a FD- $\pi$ model as long as the main purpose is to accurately represent small-signal system dynamics and potential control interactions, similar to the study in [14]. For such purposes, the accuracy of the model for very high frequencies (e.g. $\mathrm{MHz}$ ), where large deviations from the reference model must be expected, is not considered to be critical.

In order to quantify the accuracy of the cable representation, a measure of the error associated with the model must be defined. Considering the expected deviations at very high frequencies, the accuracy should be assessed within a certain frequency range. However, the frequency window where the model fulfills specific accuracy requirements should be sufficiently wide to include all the dynamics that can have an effect on the global small-signal stability of the HVDC transmission scheme.

Since the reference model for the cable is most easily expressed in the frequency domain, it is also preferable to assess the accuracy of the FD- $\pi$ model in the frequency domain. Moreover, with reference to the frequency characteristics plotted in Fig. 2, the phase error and the amplitude error should be evaluated separately.

The accuracy of the model could generally be assessed by calculating an averaged or weighted error over the frequency range of interest. However, the objective of the state-space modelling is oriented towards the assessment of stability and possible undesired behaviors. Thus, a high error in a single frequency cannot be accepted even if the average error is small. Hence, the accuracy of the model in the following parts of the paper is assessed by evaluating the maximum error in amplitude and phase within a given frequency range rather than by an averaged metric.

In order to compare the performances of several model configurations, the impedance of a cable with $n$ cascaded sections and $m$ parallel branches is referred to as $Z_{n, m}$ while the accurate frequency-dependent impedance according to Fig. 1a is defined as $Z$. These impedances also depend on the termination of the cable. Thus, the two extreme cases of short-circuited or open-ended equivalent impedances of the cable are considered in this paper. The model accuracy is then quantified by the maximum error of the impedance magnitude and angle between the FD- $\pi$ model and the reference, which is expressed as:

$$
\begin{aligned}
\epsilon_{n, m}^{a b s} & =\max _{\omega \leq \omega_{B}} 20 \log _{10}\left|Z(\omega)-Z_{n, m}(\omega)\right| \\
\epsilon_{n, m}^{a n g} & =\max _{\omega \leq \omega_{B}}\left|\angle Z(\omega)-\angle Z_{n, m}(\omega)\right|
\end{aligned}
$$

where $\omega_{B}$ is the angular frequency (in $\mathrm{rad} / \mathrm{s}$ ) corresponding to the upper frequency limit $f_{B}[\mathrm{~Hz}]$.

\section{Model accuracy EVAluation}

In general, the accuracy of an FD- $\pi$ model can widely vary depending on several factors. This section aims at providing an overview of the main factors affecting the model accuracy and to quantify their influence on the modelling of a cable with parameters as given in [17].

The accuracy of the model obviously depends on the configuration of the model itself. Indeed, the number of parallel branches and the number of cascaded pi-sections are the main factors defining the model configuration and as such both the model order and the accuracy. In general, a higher number of parallel branches and a higher number of pi-sections in series leads to a better accuracy. However, beyond a certain model order, the improvements in accuracy with further increase of the model order tend to become marginal. A more subtle effect is related to the execution of the fitting process. Indeed, the vector fitting algorithm may be fine-tuned by introducing a 


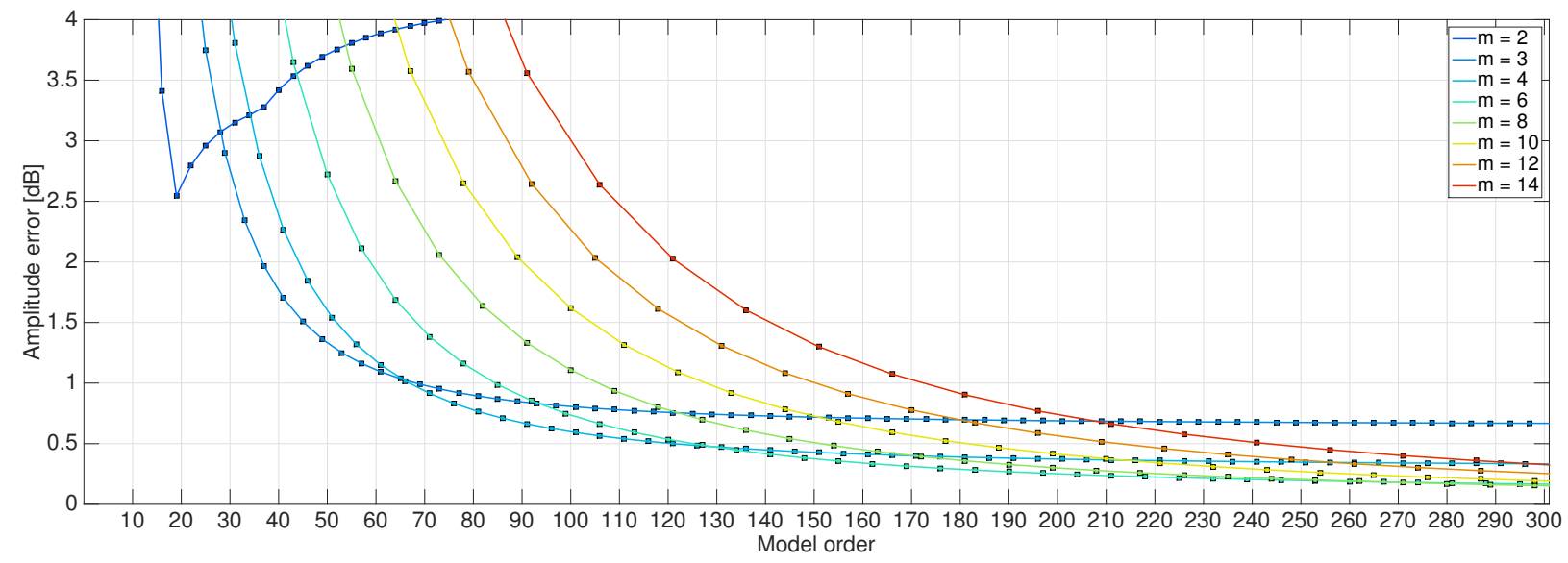

(a)

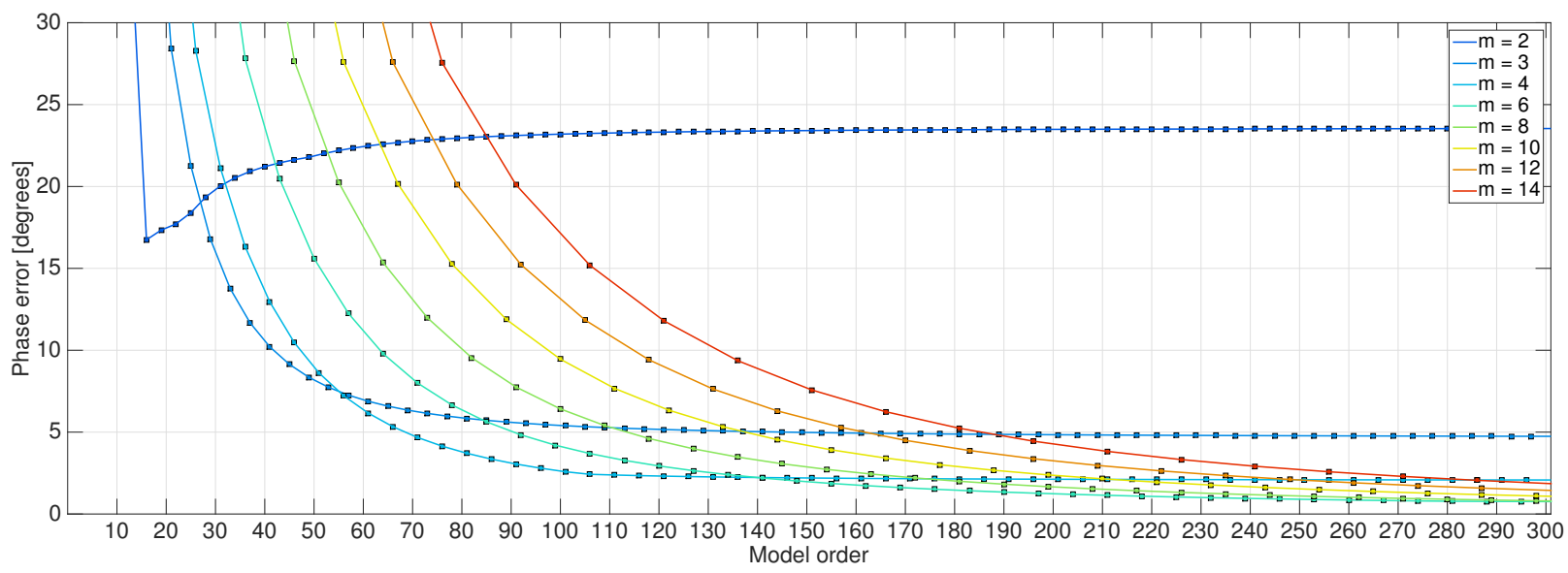

(b)

Fig. 3. Maximum amplitude error and phase error in the frequency range below $500 \mathrm{~Hz}$ as function of the model order for different number of parallel branches - short-circuited cable termination.

weighting function that can stress the accuracy in a specific frequency region [16]. This aspect is of importance if there are different requirements for the accuracy over the considered frequency range, but is considered outside the scope of this paper.

For the application in power system studies, the overall model accuracy generally depends on the characteristics of the cable and of the power system where the cable is installed. The cable length $\ell$ is also a main element affecting the frequency behavior of the cable and consequently the model accuracy. Indeed, in the the following section, a set of lengths are considered ranging from relatively short cables of $50 \mathrm{~km}$ up to long cables of $800 \mathrm{~km}$. Another critical element is the termination impedance or load impedance for the cable. This impedance can vary continuously but due to space constraints only two cases will be considered, namely an example of cable with a low impedance termination and a cable with high impedance termination. These two cases are reported separately in the following two subsections.

\section{A. Low-impedance cable termination}

As an example of the model representation in case of a cable terminated with a low impedance, the extreme case of a short-circuited cable on one end is assumed. The maximum amplitude and phase errors are shown in Fig. 3 for a $400 \mathrm{~km}$ long cable, with an upper frequency limit $f_{B}$ set to $500 \mathrm{~Hz}$. The case with $m=1$ is not shown in the figure as the error is always higher than the upper bounds set for the model to be valid. The results in Fig. 3 confirm that increasing the model order can improve the accuracy and also indicates a pattern in the accuracy associated with the configuration of the model. Indeed, both the number of pi-equivalents $n$ and parallel branches $m$ have to be increased to improve the model accuracy. It can be observed that for a given number of parallel branches $m \geq 3$, increasing the number of cascaded pisections $n$ and, thus, the order of the model, reduces the error until it reaches an asymptotic value. This asymptotic value decreases with increasing the number of parallel branches $m$. Moreover, it should be noted that the curves with lower values for $m$ cross the curves with higher values. Such crossing point define the model order $N$ where it is preferable to increase 


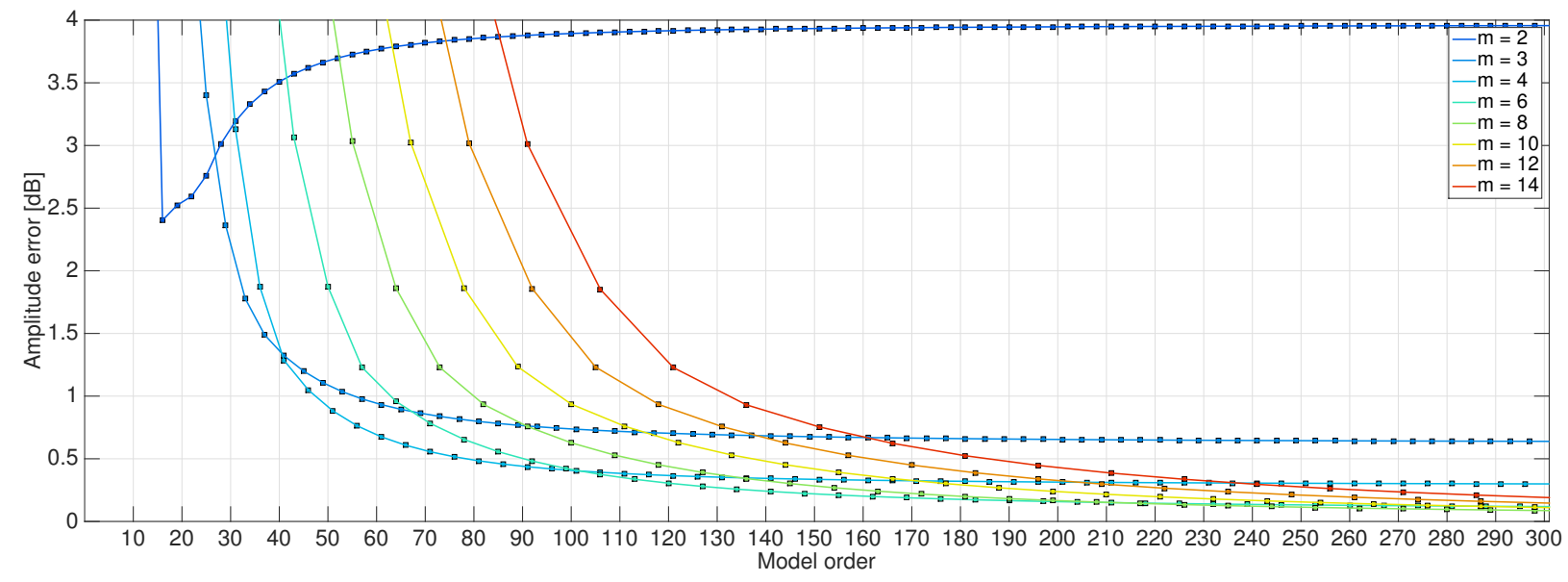

(a)

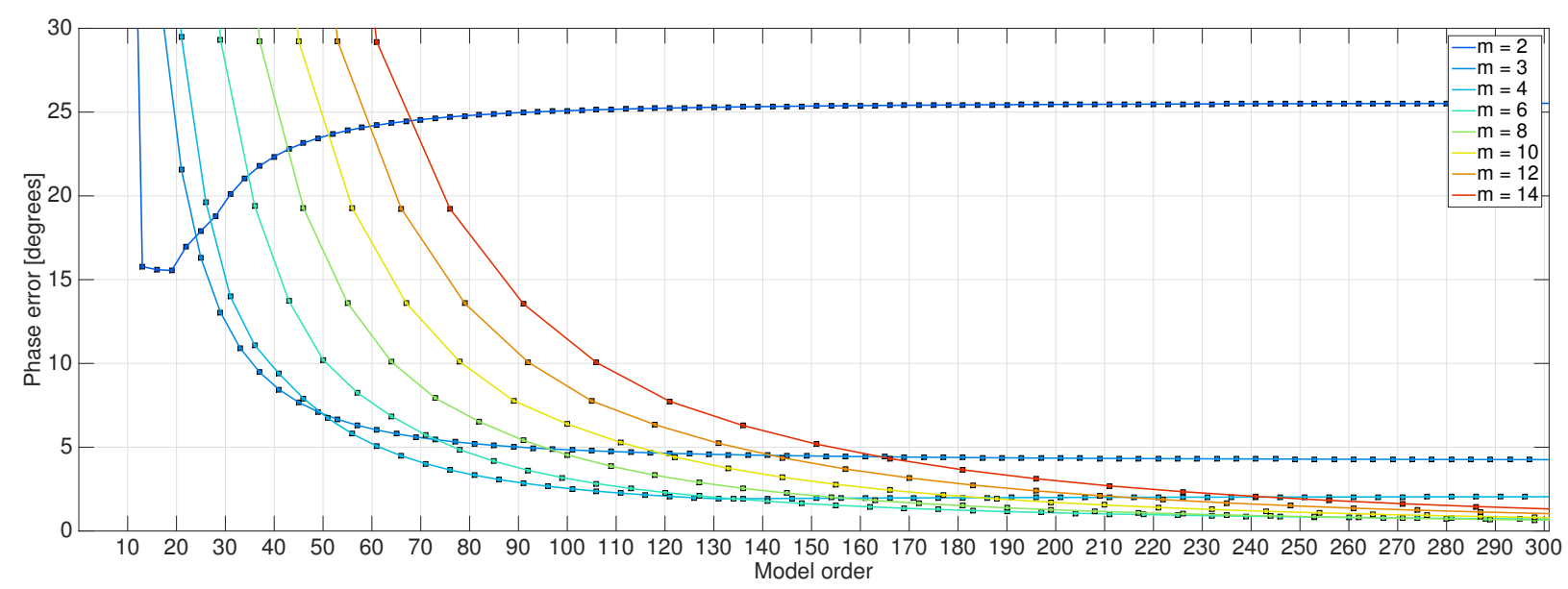

(b)

Fig. 4. Maximum amplitude error and phase error in the frequency range below $500 \mathrm{~Hz}$ as function of the model order for different number of parallel branches - open ended cable.

the number of parallel branches $m$ to obtain better accuracy. For example, from Fig. 3 it is clear that concerning the phase error, a value of $m$ equal to 4 is preferable up to an order of about 50. For model orders higher than about 55, it is clearly seen that a value of $m$ equal to 6 will provide higher accuracy both in phase and amplitude than a model with the same order and 4 of fewer parallel branches.

It can also be noticed from Fig. 3 that the special case of $m=2$ parallel branches results in a minimum error at a relatively low model order. Increasing the model order without adding more parallel branches will then increase the deviations, until the errors settle asymptotically towards relatively high values.

\section{B. High-impedance cable termination}

As an alternative configuration, a cable with a high impedance termination is considered. As a limiting case, the termination impedance is assumed to be infinite, corresponding to an open circuit on one cable end. Similarly to the previous case, the amplitude and phase error are displayed in Fig. 4. From a qualitative perspective the error plots appear rather similar to the corresponding plots with low impedance. This confirms the general trend of the error and a consistent behavior of the model. It should also be noted that the parameters for the pi-sections are the same (a pi-section with $m$ parallel branches is identical for both cases).

Comparing the results in Fig. 3 and Fig. 4, some minor differences appear between the amplitude and phase errors for the short-circuited and open ended cables. In general, the results indicate that the amplitude error is slightly higher for the case of a short circuited cable than for the open ended cable, while the phase error is highest for the open ended cable. This indicates that the actual cable termination might need to be taken into account when determining the model configuration in terms of values for $n$ and $m$ for a given model order. However, for VSC-HVDC systems with twolevel converters, a large dc-side capacitor will be present at the cable termination, which will appear as a low impedance for high frequency components. Thus, the results presented in the following will be based on the assumption of a low impedance (i.e. short circuited) cable termination. 


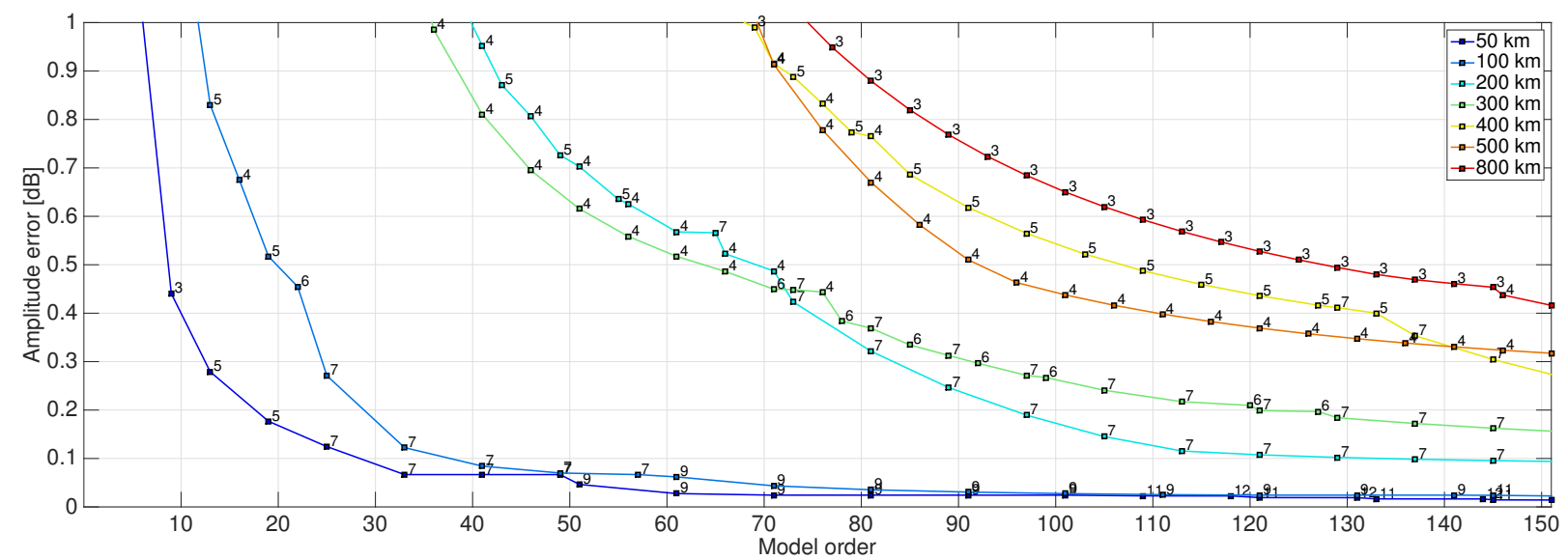

(a)

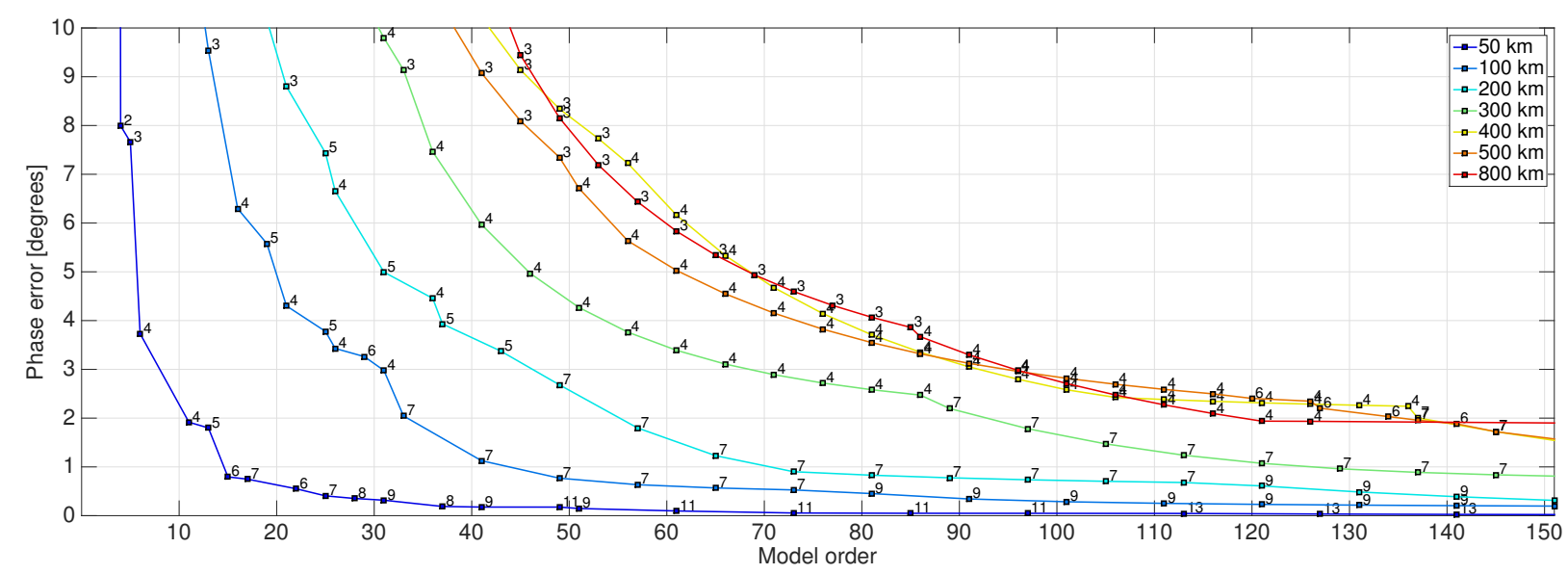

(b)

Fig. 5. Amplitude error and phase error for the optimal combination of $n$ and $m$ as function of the model order for different cable lengths.

\section{CONSIDERATIONS ON THE OPTIMAL MODEL ORDER}

The FD- $\pi$ model can be tuned according to given requirements by selecting the number of parallel branches and the number of cascaded sections. Each combination leads to a different FD- $\pi$ model, for which the accuracy can be determined as described in the previous sections. However, from a user perspective it is more natural to reverse the approach by instead specifying the minimum accuracy and the required frequency range of applicability, and determine the specifications of the model order and configuration depending on those values. Indeed, since several combinations of $n$ and $m$ will likely meet the accuracy requirements it is relevant to identify the configuration with the lowest order since it will minimize the computational efforts.

This section highlights how the optimal model order can be affected by the error tolerances imposed as maximum values for $\epsilon_{n, m}^{a b s}$ and $\epsilon_{n, m}^{a n g}$, the cable length $\ell$ and the upper frequency limit $f_{B}$. The selection of the optimal configuration is not performed by a dedicated search algorithm, but by evaluating all the possible combinations. It is expected that more efficient algorithms could be applied to identify the optimal model order under a set of requirements, but this is assumed to be outside of the scope of this paper.

As already mentioned, a low impedance termination is assumed to be most representative for a cable connected to a VSC HVDC converter terminal, although this might not necessarily ensure a conservative assessement of the model accuracy. For this reason, in the remainder of the study, the considerations are limited to a cable short-circuited at one end.

\section{A. Effect of cable length on the optimal model configuration}

A first element that can clearly affect the minimum order is the cable length $\ell$. The optimal configuration of the FD$\pi$ model for different cable lengths is reported as a function of the total model order $N$ in Fig. 5. The numbers in the vicinity of the markers report the number of parallel branches $m$ associated with this optimal configuration. It can be noticed that a longer cable in general has lower model accuracies $\epsilon_{n, m}^{a b s}$ and $\epsilon_{n, m}^{a n g}$ for the same model order $N$ or equivalently requires a higher order model order $N$ to be represented with the same degree of accuracy. Moreover, in a similar way as for traditional phasor-based pi-equivalent representation of ac transmission lines, it as can be intuitively expected that longer cables will require a higher number of pi-equivalents. Thus, the optimal model configuration for long cables will 


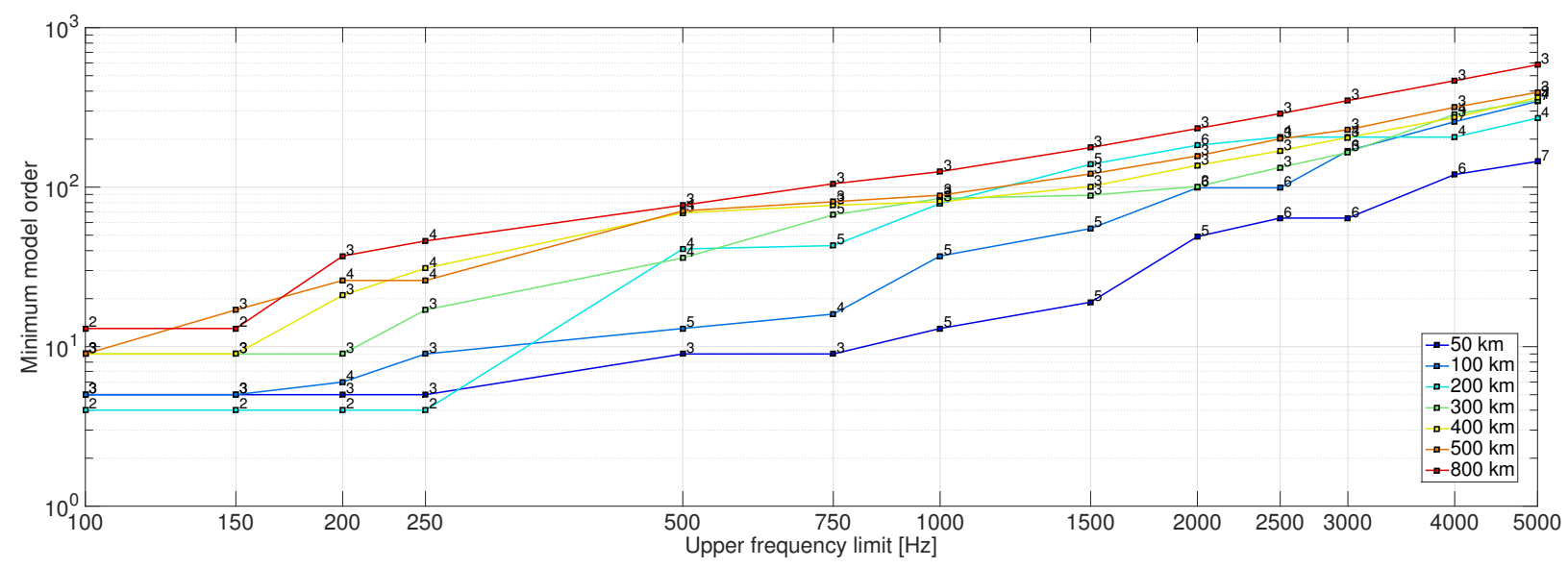

Fig. 6. Minimum model order as function of the upper frequency limit for different cable lengths.

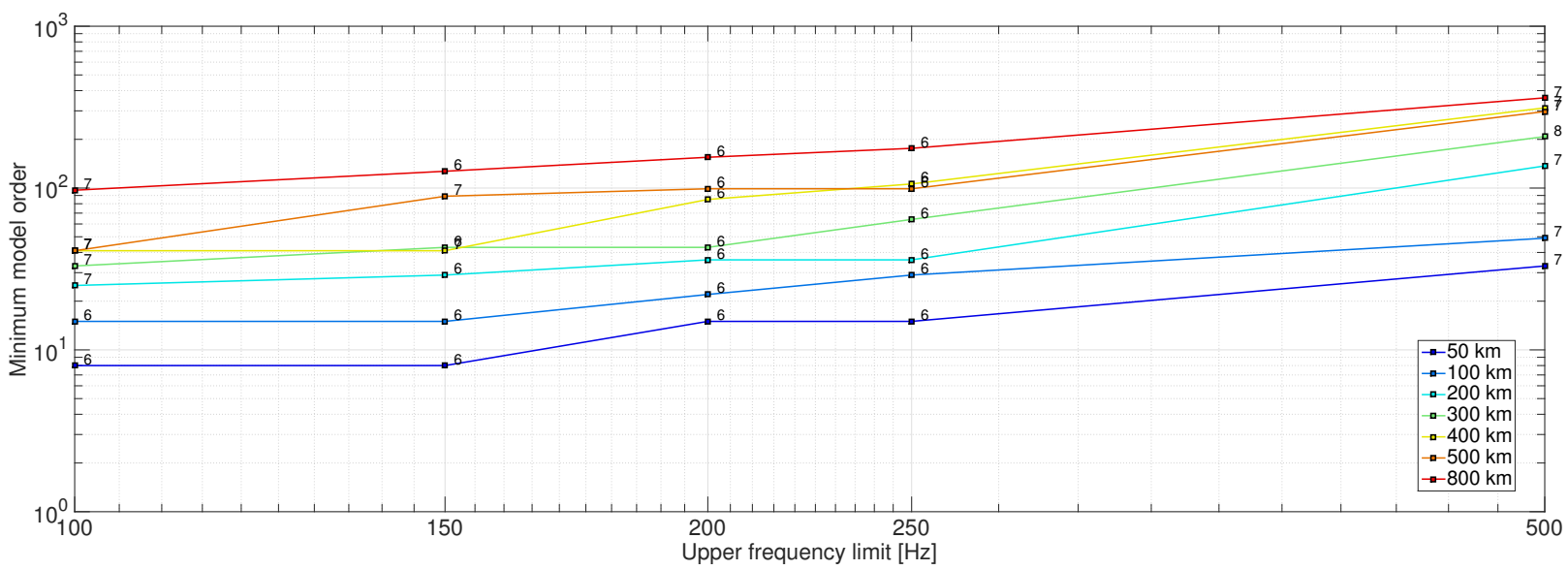

Fig. 7. Minimum model order as function of the upper frequency limit for different cable lengths (more stringent error tolerances).

require a higher number $n$ of pi-sections, implying a lower number of parallel branches $m$, for the same model order. This can be explained by the higher influence of the nonlinear hyperbolic terms in (1)-(3) for longer cable lengths, hence justifying an approximation with a higher number of pi-sections $n$. However, the results in Fig. 5 indicate that also in case of long cables it will always be necessary to use at least 3 parallel branches to obtain acceptable accuracy.

\section{B. Effect of upper frequency limit on the optimal model configuration}

The minimum model order as a function of the upper frequency limit $f_{B}$ for several cable lengths is displayed in Figs. 6-7. The value at the markers indicates the number of parallel branches for this model order. More specifically, Fig. 6 shows the evolution of the minimum model order as a function of the maximum model frequency $f_{B}$ for a tolerance on the amplitude error $\epsilon_{n, m}^{a b s}$ of $1 \mathrm{~dB}$ and a tolerance on the phase error $\epsilon_{n, m}^{a n g}$ of $15^{\circ}$. In Fig. 7, the accuracy requirements have been increased further, with a tolerance for the amplitude error $\epsilon_{n, m}^{a b s}$ of $0.1 \mathrm{~dB}$ and a tolerance on the phase error $\epsilon_{n, m}^{a n g}$ of $1^{\circ}$.
In agreement with the results presented in the previous section, the required model order increases with the cable length $\ell$. The logarithmic scale of the figures also clearly indicates that for the same constraints and the same upper frequency limit $f_{B}$, a cable of $800 \mathrm{~km}$ long would require an order approximately ten times larger than a cable of $50 \mathrm{~km}$. The model order is even more dramatically affected by the specification of the upper frequency limit $f_{B}$. Indeed, the relationship between the minimum model order $N$ and the upper frequency limit $f_{B}$ appears rather linear in the logarithmic scale of the figures, corresponding to an exponential increase in model order.

As an illustration based on the less stringent accuracy requirements applied in Fig. 6, a cable of $800 \mathrm{~km}$ requires a model order $N$ of 20 if the upper frequency limit $f_{B}$ is set to $100 \mathrm{~Hz}$. On the contrary, the minimum model order $N$ increases to over 600 for an upper frequency limit $f_{B}$ of $5 \mathrm{kHz}$ (Figs. 7). It should also be noted that in this case the increase of the model order $N$ is mostly due to a very high increase in the number of cascaded pi-sections $n$ as the parallel branches $m$ only changes from 2 to 3 . In general, the accuracy constraints have a large effect on the minimum model order, 
as noticed when comparing the two figures for the same cable length $\ell$ and upper frequency limit $f_{B}$. As a general remark, the FD- $\pi$ model can reach a satisfactory level of accuracy but the error constraints and the upper frequency limit should not be over-specified since the model order grows rather quickly. Thus, a careful consideration of the realistic needs for the model should precede the model design and optimization.

\section{CONCLusion}

The comparative study carried out in this paper shows that a model with cascaded pi-sections and multiple parallel branches (FD- $\pi$ model) can accurately represent the frequency-dependent behavior of an HVDC cable in state space form. The number of parallel branches and the number of cascaded sections are the main parameters defining the model configurations and obviously both have a major impact on the model accuracy. For the majority of cases, however, 3 or 4 parallel branches are already sufficient to reproduce the frequency dependency of the series impedance of the cable in the frequency range typically considered in studies of control interactions. Although higher numbers of parallel branches can further reduce the model errors, the improvements in terms of accuracy become less and less pronounced. Moreover, the paper discussed how the model can be configured and optimized in terms of model order once the accuracy is specified. The numerical results presented demonstrate that shorter cables generally require a lower model order than longer cables, even if the order increase is mostly due to a much higher number of cascaded pi-sections, rather than an increase in the number of parallel branches. Finally, increasing the upper frequency limit of the model gives rise to an exponential increase in the required model order for fulfilling specific accuracy requirements.

\section{ACKNOWLEDGMENT}

The authors would like to thank Bjørn Gustavsen at SINTEF Energy Research for support with the vector fitting tool.

\section{REFERENCES}

[1] N. Flourentzou, V. G. Agelidis, and G. D. Demetriades, "VSC-based HVDC power transmission systems: An overview," IEEE Trans. Power Electron., vol. 24, no. 3, pp. 592-602, 2009.

[2] P. Labra Francos, S. Sanz Verdugo, and S. Guyomarch, "New FrenchSpanish VSC link," in Proc. CIGRÉ 2012, Paris, France, Aug. 26-31, 2012, 15 pages.
[3] J. Dorn, J. Gambach, J. Strauss, T. Westerweller, and J. Alligan, "Trans bay cable - a breakthrough of vsc multilevel converters in HVDC transmission," in Proc. CIGRÉ B4 Colloquium 2012, San Francisco, CA, USA, Mar. 2012, 7 pages.

[4] G. O. Kalcon, G. P. Adam, O. Anaya-Lara, S. Lo, and K. Uhlen, "Smallsignal stability analysis of multi-terminal VSC-based DC transmission systems," IEEE Trans. Power Syst., vol. 27, no. 4, pp. 1818-1830, Nov. 2012.

[5] Y. Song and C. Breitholtz, "Nyquist stability analysis of an ac-grid connected vsc-hvdc system using a distributed parameter DC cable model," IEEE Trans. Power Del., vol. 31, no. 2, pp. 898-907, Apr. 2016.

[6] A. Morched, B. Gustavsen, and M. Tartibi, "A universal model for accurate calculation of electromagnetic transients on overhead lines and underground cables," IEEE Trans. Power Del., vol. 14, no. 3, pp. 1032 1038, Jul. 1999.

[7] N. Chaudhuri, R. Majumder, B. Chaudhuri, and J. Pan, "Stability analysis of VSC MTDC grids connected to multimachine AC systems," IEEE Trans. Power Del., vol. 26, no. 4, pp. 2774-2784, Oct. 2011.

[8] E. Prieto-Araujo, F. D. Bianchi, A. Junyent-Ferre, and O. GomisBellmunt, "Methodology for droop control dynamic analysis of multiterminal VSC-HVDC grids for offshore wind farms," IEEE Trans. Power Del., vol. 26, no. 4, pp. 2476-2485, 2011.

[9] G. Pinares, L. Tjernberg, L. A. Tuan, C. Breitholtz, and A.-A. Edris, "On the analysis of the dc dynamics of multi-terminal VSC-HVDC systems using small signal modeling," in Proc. IEEE PowerTech 2013, Grenoble, France, Jun. 16-20, 2013, 6 pages.

[10] W. Wang, M. Barnes, and O. Marjanovic, "Droop control modelling and analysis of multi-terminal VSC-HVDC for offshore wind farms," in Proc. IET ACDC 2012, Birmingham, UK, Dec. 4-6, 2012, 6 pages.

[11] W. Wang, M. Barnes, O. Marjanovic, and O. Cwikowski, "Impact of DC breaker systems on multiterminal VSC-HVDC stability," IEEE Trans. Power Del., vol. 31, no. 2, pp. 769-779, Apr. 2016.

[12] J. Beerten, S. D'Arco, and J. A. Suul, "Frequency-dependent cable modelling for small-signal stability analysis of VSC-HVDC systems," IET Gener., Transm. Distrib., vol. 10, no. 6, pp. 1370-1381, 2016.

[13] _ , "Cable model order reduction for HVDC systems interoperability analysis," in Proc. IET ACDC 2015, Birmingham, UK, Feb., 10-12 2015, 10 pages.

[14] _ , "Identification and small-signal analysis of interaction modes in vsc mtdc systems," IEEE Trans. Power Del., vol. 31, no. 2, pp. 888-897, Apr. 2016.

[15] B. Gustavsen, T. Noda, J. Naredo, F. Uribe, and J. Martinez-Velasco, Power System Transients: Parameter Determination. Taylor and Francis Group, 2010, ch. 3, pp. 137-175.

[16] B. Gustavsen and A. Semlyen, "Rational approximation of frequency domain responses by vector fitting," IEEE Trans. Power Del., vol. 14, no. 3, pp. 1052-1061, Jul. 1999.

[17] W. Leterme, N. Ahmed, J. Beerten, L. Ängquist, D. Van Hertem, and S. Norrga, "A new HVDC grid test system for HVDC grid dynamics and protection studies in EMT-type software," in Proc. IET ACDC 2015 , Birmingham, UK, Feb., 10-12 2015, 7 pages. 